\title{
BMJ Open Discovering untapped relationship potential with patients in telehealth: a qualitative interview study
}

\author{
Birgit Heckemann, ${ }^{1}$ Axel Wolf, ${ }^{2}$ Lilas Ali, ${ }^{2}$ Steffen Mark Sonntag, ${ }^{3}$ Inger Ekman ${ }^{2}$
}

To cite: Heckemann $B$, Wolf A, Ali L, et al. Discovering untapped relationship potential with patients in telehealth: a qualitative interview study. BMJ Open 2016;6:e009750. doi:10.1136/bmjopen-2015009750

- Prepublication history for this paper is available online. To view these files please visit the journal online (http://dx.doi.org/10.1136/ bmjopen-2015-009750).

Received 18 August 2015 Revised 23 December 2015 Accepted 31 December 2015

CrossMark

For numbered affiliations see end of article.

Correspondence to Birgit Heckemann; b.heckemann@ maastrichtuniversity.nl

\section{ABSTRACT}

Objectives: To explore factors that influence relationship building between telehealth professionals and patients with chronic illness over a distance, from a telehealth professional's perspective.

Design: 4 focus group interviews were conducted in June 2014. Digital recordings were transcribed verbatim and qualitative content analysis was performed using an iterative process of 3 coding rounds.

Participants: 20 telehealth professionals.

Setting: A telehealth service centre in the south of Germany that provided care for 12000 patients with chronic heart failure across Germany.

Results: Non-video telehealth technology creates an atmosphere that fosters sharing of personal information and a non-judgemental attitude. This facilitates the delivery of fair and equal healthcare. A combination of a protocol-driven service structure along with shared team and organisational values provide a basis for establishing long-term healthcare relationships. However, each contact between a telehealth professional and a patient has an uncertain outcome and requires skilful negotiation of the relationship. Although care provision was personalised, there was scope to include the patients as 'experts on their own illness' to a greater extent as advocated by person-centred care. Currently, provision of personcentred care is not sufficiently addressed in telehealth professional training.

Conclusions: Telehealth offers a viable environment for the delivery of person-centred care for patients with long-standing disease. Current telehealth training programmes may be enhanced by teaching personcentred care skills.

\section{INTRODUCTION}

The growing number of patients with noncommunicable, chronic disease and the constant increase in healthcare costs are global challenges. Health systems were originally designed to cure acute illness rather than to provide prolonged caring, monitoring or management of chronic diseases. ${ }^{1}$ Healthcare professionals require new skills and technologies to manage the shifting

\section{Strengths and limitations of this study}

- The present study may inform the current debate on integrating person-centred care principles in telehealth.

- Data collection through focus groups encouraged free discussion and exchange of opinions to mitigate 'socially desirable' comments about telehealth or person-centred care.

- We could not exclude volunteer bias as our participants received financial compensation for time spent in focus groups. However, we were able to recruit a diverse sample with a broad range of clinical and telehealth experience.

- Our sample was not designed to be statistically representative, nor can we claim reaching data saturation; however, our findings are consistent with other research on protocol-driven, nonvideo telehealth technology.

balance between acute care and the increasing numbers of patients living with a chronic disease. $^{2}$ E-Health solutions promise to deliver accessible, high-quality and costeffective remote healthcare, and play a central role in strategic healthcare planning worldwide. $^{3-5}$ E-Health is an umbrella term for all remote health information, communication and care services that are delivered and managed electronically or through information technology. ${ }^{6}$ The term 'telehealth' is situated within e-Health; more specifically: telehealth covers the remote delivery of medical and preventive care, or public health interventions via information technologies. ${ }^{7}$ In the context of chronic disease, telehealth also aims to improve patients' selfmanagement skills. There are two principal modes of telehealth delivery: video consulting and telephone (audio only) consulting. Video consulting enables the formation of good therapeutic alliances and produces similar clinical outcomes as face-to-face consultations; however, the outcomes are not superior to telephone monitoring or support. $^{8} \quad 9$ Telephone support services in 
telehealth generally combine elements of patient education, telemonitoring of symptoms and communication between healthcare staff and patients. ${ }^{9}$ Telehealth delivery is primarily protocol-driven. Patient assessment and communication between the telehealth professional (THP) and the patient are often structured by questiongenerating computer software. The combination of telephone support and remote monitoring has proven benefits with studies finding a reduction in rehospitalisation rates and mortality. Patients also reported feeling safer and becoming more knowledgeable about their disease. ${ }^{10-12}$ Telecoaching services take a more patientcentred approach; these may also be protocol-driven and facilitate monitoring and management of signs and symptoms. Telecoaching services aim to affect health behaviour, increase patient empowerment and facilitate goal setting. A recent review concluded that telecoaching significantly improves health behaviour, health status and self-efficacy in chronically ill patients. ${ }^{13}$ Despite these benefits, the effect of telehealth services on patients' health-related quality of life and well-being is limited. ${ }^{11} 13{ }^{14}$ A number of national strategies emphasise the importance of tailoring telehealth services to the needs of the individual patient. ${ }^{4}{ }^{15}$ Although telehealth services are evolving towards an increased focus on the individual patient, the emphasis often remains on symptom monitoring and control. This focus situates the current telehealth approach in the biomedical paradigm as it neglects the individual's personal experience of illness in care planning and provision. Living with a chronic disease profoundly affects a person's whole life, including social, psychological, cultural and behavioural dimensions. This personal and unique illness experience can only be captured through a patient's narrative account of living with a chronic disease, not through symptom monitoring. ${ }^{16}$ Moving towards a more comprehensive and individualised service model that considers the patients' symptoms as well as their personal illness experiences, social environment, challenges, resources and disease progression may maximise the benefits of telehealth for chronically ill patients. Person-centred care (PCC) is a concept that redresses the focus of healthcare delivery. PCC aims to actively involve the patient as an equal partner and expert on living with a chronic illness in the care planning process. ${ }^{17}$ Patients' individual resources are activated to foster their ability to self-manage their illness. Research has demonstrated that PCG reduces the experience of uncertainty in illness, improves self-efficacy and shortens hospital stays in chronically ill patients. ${ }^{18-21}$ Incorporating PCC principles in telehealth services may offer a promising model for future healthcare provisions. Although shared decision-making and egalitarian partnership are still fairly new concepts in telehealth, a recent scoping review concluded that PCC-based telehealth interventions have a positive impact on patients' quality of life and health-related quality of life. ${ }^{22}$ However, PCC telehealth services often only partially integrate PCC principles, thus leaving room for improvement. ${ }^{22}$ Currently, there is a dearth of knowledge on how more egalitarian PCC relationships between healthcare professionals and patients can be established, utilised and maintained in telehealth to enable active patient participation in decision-making and care planning. Our study addresses this knowledge gap by exploring telehealth relationships with a view to create the potential to integrate PCC principles into the care delivery for patients with chronic disease.

\section{Objective}

The present study aimed to explore factors relevant to creating and maintaining relationships between THPs and patients with chronic illness over distance, from a THP perspective.

\section{METHODS \\ Design}

The present study used qualitative content analysis of four semistructured focus group interviews with THPs. The explorative study is part of a larger research programme (registration: http://www.isrctn.com/ ISRCTN55562827) aimed at developing a telehealth service for patients with chronic heart failure (CHF) and/or chronic obstructive pulmonary disease (COPD). We chose a qualitative design to explore how relationships in telehealth are established and maintained. The focus group interviews enabled interactive discussions, diversity, reflection and development of thoughts ${ }^{23}$ among our participants about the nature of telehealth relationships.

\section{Sample}

Participants were recruited from the staff (70 staff members in total) of a service centre providing telehealth in Germany. The service centre management distributed an information letter about the study and an invitation to participate among the THPs and organised the focus group sessions. Twenty THPs agreed to take part in the study. The telehealth service centre reimbursed focus group participation as working time. Table 1 presents participants' demographics.

\section{Setting}

The participants worked in a large telehealth service centre in the south of Germany that provides care and telemonitoring for about 12000 patients with CHF across five German federal states. Patients report daily on signs, symptoms and body weight. These data are automatically transferred to the centre. To date, more than 30000 patients have participated in the telehealth programme. The telehealth service is covered by health insurance and is, therefore, free for patients. Patients and THPs never meet face-to-face; telephone calls are the exclusive means of communication. As more than 95\% of patients with $\mathrm{CHF}$ are diagnosed with at least 
Table 1 Characteristics of focus group participants $(\mathrm{N}=20)$

$\begin{array}{lc}\text { Characteristics } & \text { Number of } \\ \text { participants }\end{array}$

one concomitant chronic disease, such as hypertension, diabetes mellitus, renal failure, COPD or atrial fibrillation, the focus of the care concept is on multimorbidity. Care provision aims to strengthen self-management, prevention and early detection of deterioration through daily monitoring. The service also places strong emphasis on communication between patients and THPs to ensure and accelerate decision-making. THPs receive intensive initial and ongoing training, and performance monitoring to ensure a high standard of care provision. Figure 1 outlines the telehealth service centre's training structure.

Unless patients prefer to be cared for by one THP, a pool of three THPs is allocated to each patient so they

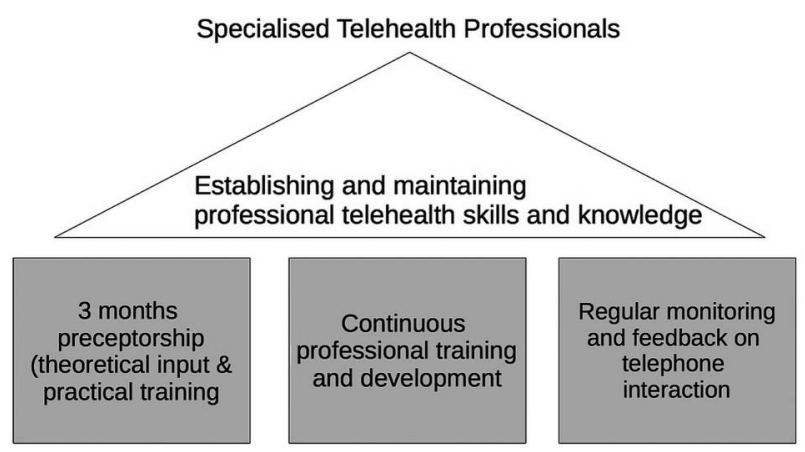

Figure 1 Training concepts for the telehealth centre: establishing and maintaining specialist telehealth skills. can benefit from a broad range of professional experience. During the initial phone call, the THPs assess factors such as the patient's mobility, nutrition, social circumstances and cognitive abilities as per the centre's protocol. Scheduled support calls, that also include teaching units, then occur every 4-6 weeks, unless the patient's condition necessitates intervention. Patient support calls and symptom monitoring are structured and protocol-driven. The THPs have a certain degree of freedom to deviate from the protocol, if necessary, for any reason. Figure 2 illustrates the telehealth service provision.

\section{Data collection and analysis}

The four focus group interviews were semistructured based on an interview guide. Table 2 outlines the topic areas and key questions discussed during the focus groups. The topic guide was developed based on a review of relevant literature and discussed among the present authors. Two of the authors (LA and AW) are telehealth experts, and four are PCC experts (IE, BH, AW and LA).

The focus group interviews were conducted on 3 and 4 June 2014. The interviews lasted between $1 \mathrm{~h} 13 \mathrm{~min}$ and $1 \mathrm{~h} 24 \mathrm{~min}$. The telehealth service centre provided a private meeting room for the focus groups. Only the interviewer and the participants were present during the interviews. The first author $(\mathrm{BH})$, a registered nurse and researcher facilitated the interviews. All interviews were digitally recorded. A professional typist transcribed the interviews according to a transcription guide. To ensure the transcript quality, the written record of the interviews was checked against the digital recording and typographical errors were corrected $(\mathrm{BH})$. The transcripts were managed with MAXQDA software for computerassisted qualitative data analysis (VERBI GmbH, Berlin, Germany). The analysis was conducted in three cycles. During the first cycle, two interviews were coded iteratively in an in vivo coding style to ground the analysis with a THP perspective. The resulting coding frame was reviewed by AW, and then revised by consensus between AW and BH. This coding frame was subsequently applied to all interviews in a second cycle, and the codes were again organised and bracketed iteratively through focused coding into a final frame of codes and categories. The categories were then condensed, connected and interpreted into themes and subthemes. An audit trail of the code lists that emerged at different stages of the coding process was generated in MAXQDA. The base unit of analysis was the sentence. Sentences in a sequence logically pertaining to particular ideas or thoughts were bracketed and coded as a single unit to preserve the original meaning. In contrast to other qualitative methodologies, such as Grounded Theory or phenomenological hermeneutics, we interpreted the interviews at a fairly superficial level, with language treated as 'a vehicle of communication, not itself an interpretive structure'. ${ }^{25}$ Basing interpretations on the 
Figure 2 Flowchart of telehealth service provision. THP, telehealth professional.

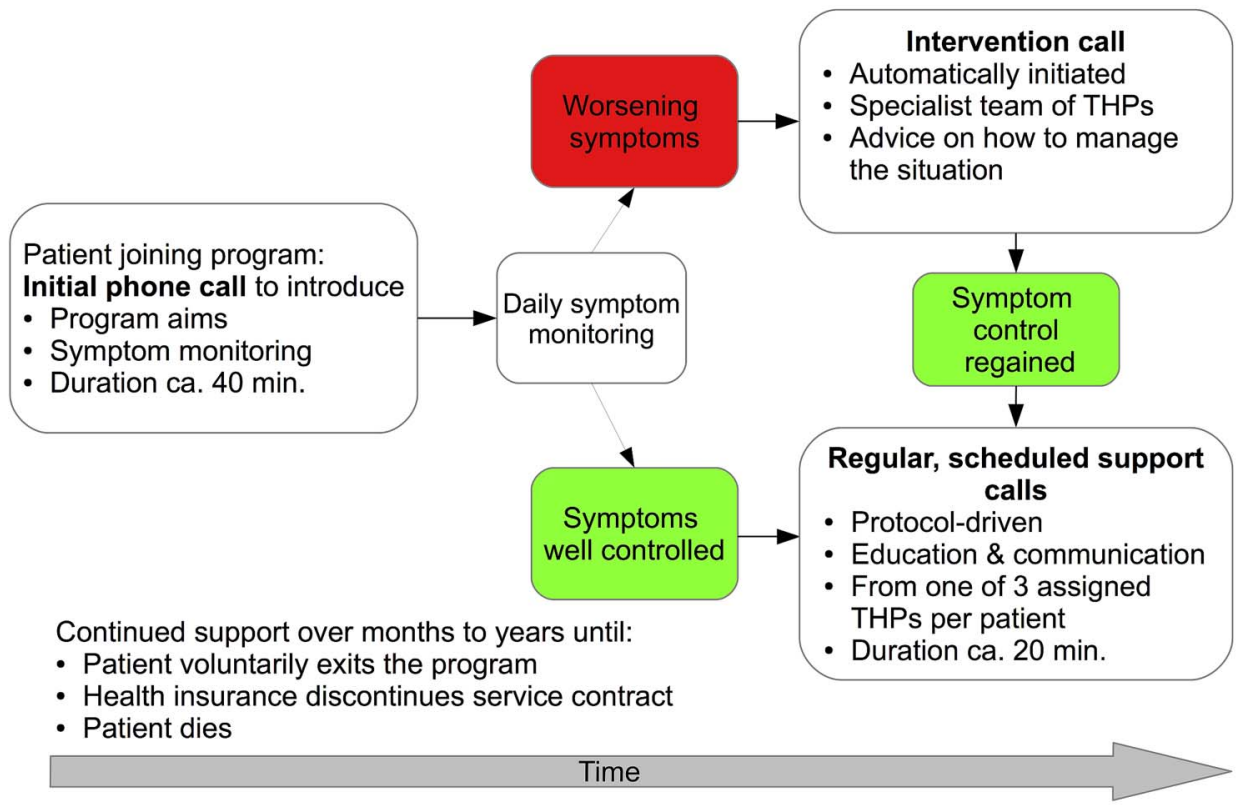

factual content of the interviews increases the transparency of our interpretations and contributes to the study's credibility and authenticity. ${ }^{25} 26$

\section{ETHICAL CONSIDERATIONS}

This present study was conducted during the preparatory phase of a larger research programme (registration:

Table 2 The interview guide: topic areas and key questions

\begin{tabular}{|c|c|}
\hline Topic area & $\begin{array}{l}\text { Key focus group discussion } \\
\text { questions }\end{array}$ \\
\hline $\begin{array}{l}\text { Patient-THP } \\
\text { interaction }^{17}\end{array}$ & $\begin{array}{l}\text { Where do you see the differences } \\
\text { in interaction with your patients } \\
\text { compared with face-to-face } \\
\text { interactions? } \\
\text { How would you describe your first } \\
\text { call to the patient? } \\
\text { What is important in telehealth } \\
\text { interaction with patients? } \\
\text { What are your techniques to } \\
\text { connect with the patient? } \\
\text { How do you win the patient's } \\
\text { trust? } \\
\text { How will you know that you have } \\
\text { established a connection with the } \\
\text { patient? } \\
\text { How do you document the } \\
\text { interaction with the patient? }\end{array}$ \\
\hline $\begin{array}{l}\text { Professional } \\
\text { identity }^{24}\end{array}$ & $\begin{array}{l}\text { Which personal qualities are } \\
\text { particularly important in } \\
\text { telehealth? } \\
\text { Which professional skills are } \\
\text { particularly important in } \\
\text { telehealth? }\end{array}$ \\
\hline
\end{tabular}

http://www.isrctn.com/ISRCTN55562827). The regional ethics committee at Gothenburg University approved the overall research programme. Since we conducted the interview study in Germany, we also submitted the study protocol to the relevant German regional ethics committee for review. The German ethics committee decided that there was no need for a formal application. Study participation was voluntary and participants were advised that they could withdraw at any point. All participants gave informed written consent. All personal information was de-identified in the interview transcripts and other documentation. We cited direct quotes from participants. The ethical issues associated with the use of direct quotes in manuscripts reporting qualitative research are complex. The deductive disclosure of participants and their potential stigmatisation are of particular concern in studies that deal with sensitive topics and/or include vulnerable participants. Our participants were not vulnerable and the topic of our study was not sensitive. We did not ask or encourage participants to disclose or discuss intimate or private information or experiences. ${ }^{27}$ The quotes we cited serve to support our findings. ${ }^{28}$ We chose quotes that reflected discussion consensus and that were sufficiently general to preclude identification of individual participants. ${ }^{29}$

\section{RESULTS}

Three themes pertaining to establishing, developing and maintaining relationships in telehealth emerged from our analysis: (1) the telehealth setting: 'challenges and facilitators'; (2) 'shared values'; and (3) 'negotiating the relationship'. Themes (1) and (2) relate to the underlying structure and culture of the telehealth environment. Theme (3) describes the transitory nature of each interaction between THP and patient. Interview excerpts are included to support our findings. 
Theme 1: the telehealth setting: intrinsic challenges and facilitators

This theme related to the particular challenges and facilitators associated with the telehealth setting. The THPs emphasised how 'not seeing' a patient altered the personal and professional experience of care provision.

\section{Professional skills, knowledge and personal qualities}

'Not-seeing' complicated patient assessment. THPs relied entirely on information verbalised by the patient, with no opportunity to support or authenticate this through their own observations. THPs counterbalanced this disadvantage with attentive listening and use of sophisticated communication techniques. They reported focusing on the patient's verbal expression and described being highly sensitive to any changes in the tone and pitch of a patient's voice. In addition to advanced listening and communication skills, THPs emphasised the importance of extensive professional clinical experience and medical knowledge, combined with personal qualities such as having a pleasant voice and being open-minded.

\section{Non-judgemental attitude}

THPs reported that 'not seeing' resulted in a less judgemental attitude in both partners in the dyad. 'Looks' did not influence feelings of sympathy or antipathy, and care provision was perceived as being fairer. Combined with the anonymity of telephone contact, the telehealth environment enabled patients to share even private information:

IV1, THP 2: [...] this anonymity, paradoxically, creates a certain closeness, because patients open up in a different way. They tell you really intimate details of their lives and that has something of a confessional character, because they cannot see you. [...] they can get rid of their worries and woes $[\ldots]$.

Overall, THPs regarded the absence of visual clues in telehealth as an advantage and facilitator to relationship building.

\section{Telehealth structure}

Telehealth calls are one-on-one appointments between THPs and patients. The THPs appreciated this and described this as a facilitator, particularly when compared with a hospital setting, where care staff must often respond to numerous demands at any one time. The telehealth service centre's documentation protocol also facilitated relationship building, prompting THPs to record extensive factual information about patients' lives, physical and psychological state and social situation. This patient record enabled THPs to prepare themselves well for each support call.
Theme 2: shared team and organisational values

THPs across all focus groups spoke at length about the values they shared across the team.

\section{Human dignity}

THPs emphasised the importance of engaging with the patient in a respectful manner and providing holistic personalised care. Specifically, this involved 'meeting' patients where they were in terms of phase of life, needs, and cognitive and linguistic abilities. THPs reported they adapted their language to the patients' needs to maximise patients' understanding of the information provided.

\section{Enabling and autonomy}

Increasing self-efficacy (ie, the ability to self-monitor and communicate signs and symptoms), and motivating and educating the patient were considered to be important. All THPs believed that all the patients were capable people. They aspired to enable patients to unlock their innate potential by making suggestions or giving advice. However, they accepted that the final decisions about whether to follow advice always remained with the patient:

IV1, THP 1: I think the difference [between our telehealth service and other healthcare providers] is that with us, patients do not HAVE to [do certain things] and they are not constantly being told what they CANNOT do. $[\ldots]$.

\section{Prioritising the patient's needs}

The THPs reported prioritising an individual patient's needs over protocol adherence if they realised that patients were experiencing difficult life situations:

IV1, THP 4: If there is sometimes something very dramatic [happening in the patient's life] then we let the heart be the heart, and if there was a quarter of an hour scheduled [for the phone call] then we may take half an hour. This is an exception, but there are situations when other things [than the heart condition] are more important. And then we listen. [...] When somebody has died [...]. I am not very interested [in hearing] if [the patient's] legs are swollen.

All of these values were supported by the telehealth service centre's organisational culture, which empowered THPs to deviate from the set protocol if they felt this to be in the patient's best interest. The THPs could, therefore, spend more time with a patient in need and less time with patients who did not require much support.

\section{Theme 3: negotiating the relationship}

The THPs stressed that relating to and connecting with patients was essential to delivering telehealth. The relationship between THPs and patients developed two trajectories: a 'macrotrajectory' and a 'microtrajectory'. 
During each support call, the patient and the THP established and negotiated a relationship on a microtrajectory. Successful relationship building at this level, in addition to daily telemonitoring, enabled the macrotrajectory or the relationship between the service provider and patients over the course of several years.

\section{The microtrajectory}

The THPs highlighted that the first telephone call was an important milestone at both the microrelationship and macrorelationship level. This was the stage where patients decided whether or not to join the programme. Gaining the patient's trust in the service was therefore crucial at this point. THPs indicated that while some patients were interested and easy to engage with from the start, others were more doubtful. Some THPs reported that particularly sceptical patients challenged their professional knowledge. The THPs felt that the more they personally connected with a patient at this early stage, the better the long-term relationship thrived. However, all telephone calls-initial as well as regular support calls-required careful negotiation and the course of each interaction remained unpredictable. Each interaction could be influenced by the patient's current personal circumstances and mood or preferences. The THPs' likened the relationship management to a dance. In this 'dance', the THPs constantly strived to establish a connection with the patient. THPs reported adapting their behaviour, choice of words and tone of voice to what they felt worked best to engage with each patient. They had to be self-aware and manage their own emotional reaction to the patient. This could also entail distancing themselves from highly emotional patients:

IV3, THP 4: [...] you just stay calm and keep an open mind. I had the experience that when somebody grumbles on the phone [...] and they are initially aggressive and just have to get rid of all this [aggression], then I let them talk and talk and talk and at some point [the patient says]: "that was stupid, wasn't it? That did not have anything to do with you" and then I say: "That's okay, are you feeling better?"

THPs reported other signs of a successful connection such as the patient being engaged, talking freely, asking questions, thanking the THPs for the telephone call or asking for particular topics to be covered in the next appointment. THPs also described feeling the connection. Others described entering a flow in the dialogue and feeling emotionally very close to the patient, or that the chemistry between them and the patient was just right. However, these feelings could be deceptive:

IV4, THP 3: There are [...] patients, who say good-bye, make arrangements for the next call, ask for a topic. Then you know for certain, this was ok. And normally, I don't put down the receiver first, but I always wait until the patient hangs up. There are some [patients] where you have the feeling "this was a really great conversation" and then your hear "Uhh, boah" [sighing or groaning noises from the patient]. And then you know [...]. My perception was wrong [...] Sometimes you get this reaction which is not positive. [...].

\section{The patient's narrative}

The patient's narrative is a personal story of their experience with their illness. The THPs reported that listening to the patient's narrative was important for the relationship building. They encouraged patients to share their story if the account was directly related to the illness. When listening to the narrative, the THPs reported actively picking up on 'anchor words or phrases' and using these to guide the conversation. The overall aim of the conversation appeared to be to elicit factual information about the illness, and to provide suggestions and professional advice accordingly. THPs regarded themselves as healthcare experts on the disease.

These three themes describe the cornerstones for establishing relationships between THPs and chronically ill patients in the telehealth setting. Two themes related to the organisational structure and values that define this particular setting, and one theme related to a more transitory aspect of relationship building, characterised by the constant creation and reaffirmation of the THPpatient relationship. All three themes enabled a 'macrotrajectory' or patients' long-term relationships with the telehealth service. The TPHs reported that with the passing of time, patients grew accustomed to telephone communication with the THPs. Trust was maintained and increased. Depending on cognitive and linguistic capabilities, patients also acquired competencies, for example, learnt medical terms to describe their disease, and associated signs and symptoms. At a macro level, interactional routines became established as patients were socialised into the telehealth programme over time. An overview of the final coding frame and interpretation into themes is set out in figure 3 .

\section{DISCUSSION}

\section{Principal findings}

Our study explored the creation, development and maintenance of telehealth relationships from a THP's perspective. THPs reported that the non-video telehealth service environment created an atmosphere in which patients feel confident about sharing intimate or private health information. It also promoted a nonjudgemental attitude that facilitated fair and equal healthcare treatment for all individuals. However, patient assessment was hampered by the absence of visual clues. The combination of a protocol-driven service structure, and shared team and organisational values provided the foundation for establishing long-term THP-patient relationships, although each telephone contact with a patient remained unpredictable. THPs used their professional and personal knowledge and skills to renegotiate the relationship, and educate and assess the patient. The 
Code

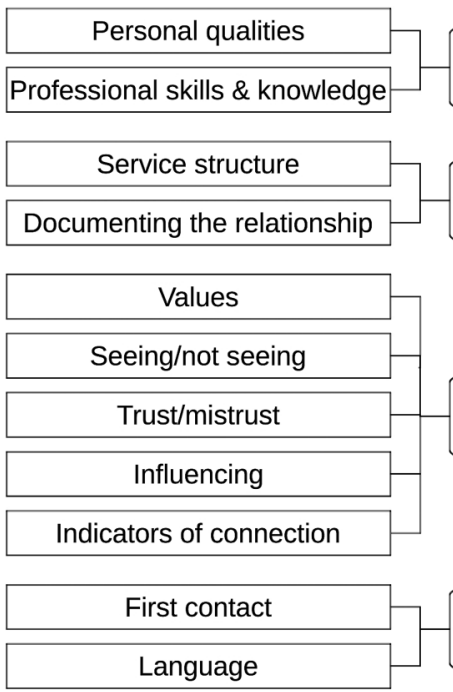

Category

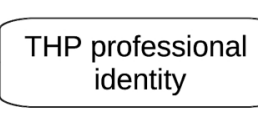

Telehealth service
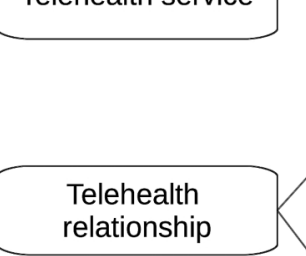

relationship

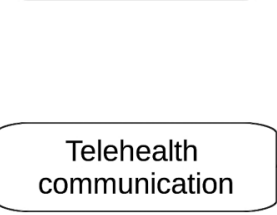

Theme

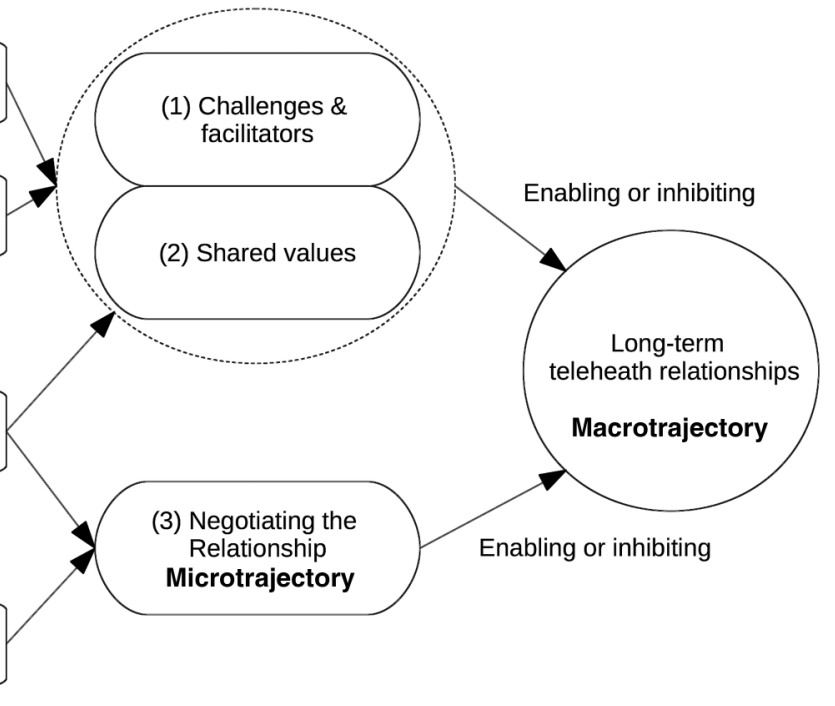

Figure 3 The final coding tree: codes, categories and themes. THP, telehealth professional.

patient's narrative was particularly important in eliciting factual information about their condition. THPs saw themselves as key people who solved problems and provided advice to the patient. Protocol-driven, structured telephone support is the most common approach for providing telehealth services to patients with CHF and effective in reducing disease-related rehospitalisation and mortality. ${ }^{10}$ Patients reported being satisfied and feeling cared for with regular telemonitoring. ${ }^{30}$ However, THPs have expressed concern that frequent telemonitoring may lead to increased patient dependency on telehealth services. ${ }^{31}$ Two recent scoping reviews on chronic care management related to PCC found that current e-Health provision remains primarily focused on telemonitoring, concluding that despite the current international drive towards patient participation and shared decision-making in healthcare planning, patients are still regarded as passive recipient of care in telehealth. ${ }^{22}{ }^{32}$ Staff may also perceive a strictly protocoldriven approach to be at odds with carers' professional humanistic and holistic values. ${ }^{24} 223032$ The THPs in our study negotiated the proposed juxtaposition between protocol adherence and professional practice with relative ease. They felt empowered to adapt the telehealth service centre protocol to the individual patient's needs. Unscripted, personalised telecoaching that focuses on patients' personal priorities and motivations has been shown to increase health behaviour and selfefficacy in chronically ill patients. ${ }^{12}$ However, neither telecoaching nor telemonitoring lead to an increased quality of life in patients with chronic disease. ${ }^{11} 1314$ Overall, the THPs felt that the protocol-driven service structure combined with unscripted, personalised coaching, and their holistic values and professional skills promoted relationship building and care provision. This finding highlights the importance of achieving a good balance between protocol-driven structures and holistic care values in telehealth to successfully build relationships. ${ }^{13}$ However, the THPs, as healthcare experts, aimed to provide the patient with information and support. The current format of telehealth monitoring and coaching affirms that the patient is in the role of a more or less passive recipient of care. ${ }^{16}$ Including more unscripted person-centred, flexible approach that caters to the individual patients' needs may increase the benefits gained from telehealth services.

There may be untapped potential in current practice that could be exploited by fully integrating a PCC approach, one that considers the patient as an expert on their disease and equal partner in shared care planning. ${ }^{1617} 21$

\section{Strengths and limitations}

Healthcare organisations worldwide are pushing the integration of PCC principles in telehealth. How this will be best achieved is a matter of current debate. Our findings may substantially inform this debate. The qualitative design is a particular strength of the present exploratory study as it uncovered the importance and possibility of balancing a protocol-driven service approach with a personalised care approach through shared organisational values. In our focus groups, we encouraged free discussion and exchange of opinions by using a format that minimised 'socially desirable' comments about telehealth or PCC. Although the provision of financial compensation for time spent in focus groups may have resulted in volunteer bias, it served as an incentive and helped to recruit a diverse sample of THPs with a broad range of clinical and telehealth experience. Our sample was not designed to be statistically representative nor can we claim reaching data saturation; however, our findings that the telehealth environment fosters mutual respect and sharing of personal information are 
consistent with previous research on protocol-driven, non-video telehealth technology. ${ }^{24} 33$

\section{Implications for future research}

Our results indicate that there is untapped potential in the relationship between patients and THPs in the telehealth and telecoaching domain. Patients can be activated and engaged to a greater extent in shared decision-making and care planning. This requires communication and feedback loops between patients and THPs, as well as awareness of enhanced power sharing, and staff and patient education. ${ }^{34}$ With the current focus on telemonitoring in telehealth, further research should focus on redressing this balance by developing supplementary training elements for THPs to support the integration of PCC into telehealth. The approach of the Gothenburg Centre for Person-Centred Care (gPCC) comprises three processes: (1) initiating a partnership through the patient narrative, (2) working the partnership through shared decision-making, and (3) safeguarding the partnership by documenting the patient narrative. This approach may be a suitable basis for integrating PCC in telehealth. ${ }^{17} 21$

\section{CONCLUSION}

Long-term relationships between THPs and patients with a chronic illness can be created, developed and maintained over a distance. A protocol-driven service structure, and shared team and organisational values support this process. THPs' professional skills and personal qualities in engaging with the patients' narratives affirm the relationship during each telephone contact. However, there is potential to activate patients to become equal partners in care planning and shared decision-making to a greater extent. Integrating PCC principles in telehealth and telemonitoring may maximise the potential of telehealth technology and enable patients with a chronic illness to manage their lives more independently. Extended theories on PCG delivery over a distance need to be developed in order to achieve this. PCG interaction theories should also inform the development of training on person-centred communication, interaction and care delivery to supplement and enrich current specialist training programmes for THPs.

\author{
Author affiliations \\ ${ }^{1}$ CAPHRI, School for Public Health and Primary Care, UM/CAPHRI, \\ Maastricht, The Netherlands \\ ${ }^{2}$ The Gothenburg Centre for Person-Centred Care (GPCC), Institute of Health \\ and Care Sciences, Sahlgrenska Academy, University of Gothenburg, \\ Gothenburg, Sweden \\ ${ }^{3}$ Health Care Systems GmbH (HCSG), Bavaria, Munich, Germany
}

Acknowledgements The authors are grateful to all the healthcare professionals who participated in this study for sharing their experiences and thoughts generously, and for engaging so enthusiastically in the focus group discussions.

Contributors BH, AW, LA, SMS and IE contributed to the conception or design of the work. BH, AW, LA and IE contributed to the acquisition, analysis, or interpretation of data; the drafting and critical revision of the manuscript; and to the provision of important intellectual content. BH, AW, LA, SMS and IE approved of the final version to be published and agreed to be accountable for all aspects of the work in ensuring that questions related to the accuracy or integrity of any part of this manuscript are appropriately investigated and resolved.

Funding This research received no specific grant from any funding agency in the public, commercial or not-for-profit sectors. It received non-financial support from the Gesellschaft für Patientenhilfe DGB mbh (GPH), Luise-Ullrich-Str. 4, 82031 Grünwald, Germany.

Competing interests None declared.

Patient consent Obtained.

Ethics approval Regional Ethical Review Board, Gothenburg, Sweden (application Nr 687-14). Bayerische Landesärztekammer (Regional ethics committee) Munich, Bavaria (no formal application required).

Provenance and peer review Not commissioned; externally peer reviewed.

Data sharing statement No additional data are available.

Open Access This is an Open Access article distributed in accordance with the Creative Commons Attribution Non Commercial (CC BY-NC 4.0) license, which permits others to distribute, remix, adapt, build upon this work noncommercially, and license their derivative works on different terms, provided the original work is properly cited and the use is non-commercial. See: http:// creativecommons.org/licenses/by-nc/4.0/

\section{REFERENCES}

1. Potter SJ, McKinlay JB. From a relationship to encounter: an examination of longitudinal and lateral dimensions in the doctor-patient relationship. Soc Sci Med 2005;61:465-79.

2. World Health Organization. Preparing a health care workforce for the 21 st century. The challenge of chronic conditions. Geneva, Switzerland, 2005

3. World Health Organization. mHealth: new horizons for health through mobile technologies: second global survey on eHealth Geneva, Switzerland: World Health Organization, 2011.

4. Stroetmann K, Aartmann J, Stroetmann V, et al. European countries on their journey towards national eHealth infrastructures. Final European progress report. Brussels, Belgium, 2011.

5. World Health Organization. National eHealth Strategy Toolkit. Geneva, Switzerland: World Health Organization, 2012.

6. Royal College of Nursing. eHealth: the future of health care. Royal College of Nursing position statement. London, UK: Royal College of Nursing, 2012

7. Doarn CR, Pruitt S, Jacobs J, et al. Federal efforts to define and advance telehealth-a work in progress. Telemed $J$ E Health 2014;20:409-18.

8. Steel K, Cox D, Garry H. Therapeutic videoconferencing interventions for the treatment of long-term conditions. J Telemed Telecare 2011;17:109-17.

9. Wootton R. Twenty years of telemedicine in chronic disease management-an evidence synthesis. J Telemed Telecare 2012;18:211-20.

10. Inglis SC, Clark RA, McAlister FA, et al. Which components of heart failure programmes are effective? A systematic review and meta-analysis of the outcomes of structured telephone support or telemonitoring as the primary component of chronic heart failure management in 8323 patients: abridged Cochrane review. Eur J Heart Fail 2011;13:1028-40.

11. Kenealy TW, Parsons MJ, Rouse AP, et al. Telecare for diabetes, CHF or COPD: effect on quality of life, hospital use and costs. A randomised controlled trial and qualitative evaluation. PLOS ONE 2015;10:e0116188.

12. Steventon A, Bardsley M, Billings J, et al. Effect of telehealth on use of secondary care and mortality: findings from the Whole System Demonstrator cluster randomised trial. BMJ 2012;344:e3874.

13. Dennis SM, Harris M, Lloyd J, et al. Do people with existing chronic conditions benefit from telephone coaching? A rapid review. Aust Health Rev 2013;37:381-8.

14. Cartwright M, Hirani SP, Rixon L, et al. Effect of telehealth on quality of life and psychological outcomes over 12 months (Whole Systems Demonstrator telehealth questionnaire study): nested study of patient reported outcomes in a pragmatic, cluster randomised controlled trial. BMJ 2013;346:f653. 
15. APS Group Scotland. A National Telehealth and Telecare. Delivery plan for Scotland to 2015. Driving improvement, integration and innovation. Edinburgh, UK: Scottish Government by APS Group Scotland, Crown copyright 2012, 2012.

16. Engel GL. The need for a new medical model: a challenge for biomedicine. Science 1977;196:129-36.

17. Ekman I, Swedberg K, Taft C, et al. Person-centered care-ready for prime time. Eur J Cardiovasc Nurs 2011;10:248-51.

18. Dudas K, Olsson LE, Wolf A, et al. Uncertainty in illness among patients with chronic heart failure is less in person-centred care than in usual care. Eur J Cardiovasc Nurs 2013;12:521-8.

19. Ekman I, Wolf A, Olsson LE, et al. Effects of person-centred care in patients with chronic heart failure: the PCC-HF study. Eur Heart $J$ 2012;33:1112-19.

20. Fors A, Ekman I, Taft C, et al. Person-centred care after acute coronary syndrome, from hospital to primary care-a randomised controlled trial. Int J Cardiol 2015;187:693-9.

21. Ekman I, Hedman H, Swedberg K, et al. Commentary: Swedish initiative on person centred care. BMJ 2015;350:h160.

22. Wildevuur SE, Simonse LW. Information and communication technology-enabled person-centered care for the "big five" chronic conditions: scoping review. J Med Internet Res 2015;17.

23. Hennink P, Leavy P. Understanding focus group discussions. New York, USA: Oxford University Press, 2014.

24. Snelgrove SR. Nursing work in NHS Direct: constructing a nursing identity in the call-centre environment. Nurs Inq 2009; 16:355-65.
25. Sandelowski M. Whatever happened to qualitative description? Res Nurs Health 2000;23:334-40.

26. Whittemore R, Chase S, Mandle C. Pearls, pith, and provocation. Validity in qualitative research. Qual Health Res 2001;11: 522-37.

27. Kaiser K. Protecting respondent confidentiality in qualitative research. Qual Health Res 2009;19(11):1632-41.

28. Corden A, Sainsbury R. Using verbatim quotations in reporting qualitative social research: Researchers' views. 2006. https://www. york.ac.uk/inst/spru/pubs/pdf/verbquotresearch.pdf (accessed 18 Oct 2015).

29. Hollander J. The social contexts of focus groups. J Contemp Ethnogr 2004;33:602-37.

30. Riley JP, Gabe JP, Cowie MR. Does telemonitoring in heart failure empower patients for self-care? A qualitative study. J Clin Nurs 2013;22:2444-55.

31. Fairbrother $\mathrm{P}$, Ure $\mathrm{J}$, Hanley $\mathrm{J}$, et al. Telemonitoring for chronic heart failure: the views of patients and healthcare professionals-a qualitative study. J Clin Nurs 2014;23:132-44.

32. Gammon D, Berntsen GK, Koricho AT, et al. The chronic care model and technological research and innovation: a scoping review at the crossroads. J Med Internet Res 2015;17:e25.

33. Oudshoorn N. Telecare technologies and the transformation of healthcare. New York, USA: Palgrave MacMillan, 2011.

34. Longtin Y, Sax H, Leape LL, et al. Patient participation: current knowledge and applicability to patient safety. Mayo Clin Proc 2010;85:53-62. 